\title{
Use of the pictorial Sleepiness and Sleep Apnoea Scale in Chinese patients with suspected obstructive sleep apnoea syndrome
}

\author{
Yang Ji ${ }^{1}$, Hongxia Wang ${ }^{1}$, Min Liu ${ }^{1}$, Martyn R. Partridge ${ }^{2}$ \\ ${ }^{1}$ Division of Respiratory Medicine, University of Hong Kong-Shenzhen Hospital, Shenzhen, China; ${ }^{2}$ National Heart and Lung Institute, Imperial \\ College London, UK \\ Contributions: (I) Conception and design: Y Ji, MR Partridge; (II) Administrative support: Y Ji; (III) Provision of study materials or patients: Y Ji, H \\ Wang, M Liu; (IV) Collection and assembly of data: Y Ji, H Wang, M Liu; (V) Data analysis and interpretation: All authors; (VI) Manuscript writing: \\ All authors; (VII) Final approval of manuscript: All authors. \\ Correspondence to: Dr. Yang Ji. Division of Respiratory Medicine, The University of Hong Kong-Shenzhen Hospital, 1, Haiyuan 1st Road, Futian \\ District, Shenzhen, China. Email: jiy@hku-szh.org.
}

\begin{abstract}
Background: Sleep related breathing disorders represent a significant health burden. Being able to stratify patients according to their need for differing types of investigation and predicting the likelihood of obstructive sleep apnoea syndrome (OSAS) is helpful. This study attempts to assess the value of a pictorial Sleepiness and Sleep Apnoea Scale (pSSAS) in this process.

Methods: A total of 126 sequential patients attending a sleep service with suspected OSAS completed the pSSAS, the Epworth Sleepiness Score (ESS), and the Berlin Questionnaire (BQ) prior to full polysomnography.

Results: With Apnoea-hypopnea index (AHI) $>15$ as the positive diagnostic criterion, the area under the receiver operating characteristic curve (AUC) of the BQ was the highest (0.683), followed by pSSAS and ESS (AUC 0.648 and 0.516, respectively). With AHI $>30$ as the positive diagnostic criterion, the AUC of pSSAS was the highest (0.696), followed by BQ and ESS (AUC 0.653 and 0.510 , respectively). With $\mathrm{MiniSO}_{2}<80 \%$ as the positive diagnostic criterion, the AUC of pSSAS was the highest (0.736), followed by BQ and ESS (AUC 0.634 and 0.516 , respectively).

Conclusions: This study shows that the pSSAS which was first tested in a European population performs equally well amongst a Chinese population. The pSSAS performed in a similar fashion to the BQ in predicting those likely to have OSAS and was superior at predicting those who have severe OSAS. Because it is a pictorial questionnaire, it has advantages for those who may have reduced health literacy, a problem which is under-recognized in most healthcare systems.
\end{abstract}

Keywords: Screening; sleep disorders; health literacy

Submitted Jun 07, 2020. Accepted for publication Aug 19, 2021.

doi: $10.21037 /$ jtd-20-2152

View this article at: https://dx.doi.org/10.21037/jtd-20-2152

\section{Introduction}

The health burden associated with ventilatory abnormalities during sleep is considerable. The commonest problem, obstructive sleep apnoea syndrome (OSAS), represents a significant challenge to health systems globally. Previously promoted gold standard investigations such as full polysomnography (PSG) are unlikely to be available to all and simpler methods of investigation have been shown to give equivalent results (1). There are similar reports of equivalent results being achieved by services offering diagnostic investigations and treatment in the community as opposed to within specialist hospital sleep services $(2,3)$.

In developing such services, it is essential that patients are correctly channelled so that those at highest risk are seen promptly and that those with more complicated 
conditions attend the more specialist services. No single screening test or management pathway has been recommended but there are widely used tools to assess the cardinal feature of sleepiness (4), and scoring systems which have been suggested to indicate a high probability of a patient having OSAS (5-7). One difficulty with some of these questionnaires is that self-completion may be difficult for those with impaired health literacy and the size of this problem may be very significant and underestimated in both general medical practice (8) and specifically amongst those attending sleep services (9).

Recently a pictorial Sleepiness and Sleep Apnoea Scale (pSSAS) has been investigated in the United Kingdom and in Switzerland and shown to be capable of correctly identifying patients with a high probability of having OSAS (10). It is not known whether such scales, which include images reflecting body size and neck circumference, are equally valid in a Chinese population where other morphological characteristics may contribute to OSAS, but a recent Chinese study using the NoSAS score, which also includes questions regarding Body Mass Index (BMI) and neck circumference, suggests such information is probably equally relevant in a Chinese population (11).

We present the following article in accordance with the STARD reporting checklist (available at https://dx.doi. org/10.21037/jtd-20-2152).

\section{Methods}

\section{Study design and patients}

A total of 152 sequential patients attending a specialist sleep service at University of Hong Kong-Shenzhen Hospital, Shenzhen, China with suspected OSAS were recruited for study and underwent investigation and completed the relevant questionnaires from January 2018 to April 2019. It was subsequently shown that 26 of these patients had some missing data and could not be included [19 missing a value in pSSAS, 15 in ESS, and 15 in Berlin Questionnaire (BQ)] leaving 126 patients with complete data including the pSSAS and the BQ (6). The English and Swiss German versions of the pSSAS can be found in the article by Edelmann and colleagues (10) and a copy of the English version is shown in Figure S1. A copy of the Chinese version of the pSSAS used in this study may be requested from the corresponding author. Three Chinese native speakers with very good knowledge of the English language independently translated the text of the English version of the pSSAS. The individual translations were then reviewed and differences in translation were addressed at a consensus meeting and an easily understandable style and wording was chosen that reflects the meaning of the original version as closely as possible. In the Chinese version, a third answer option "don't know" represented by a question mark besides "breathing normally when asleep" and "stopping breathing when asleep" for question number 8 was added as this had been shown to be helpful in the Swiss German version of the pSSAS. The validated, Chinese language version of the ESS was used to measure excessive daytime sleepiness (12). The sleep nurses instructed the subjects to finish the three questionnaires including pSSAS. This study has documented review and approval from the University of Hong Kong-Shenzhen Hospital Institutional Review Board (approval number [2019]092) and is conducted in accordance with the Declaration of Helsinki (as revised in 2013). Written informed consent have been provided by the participants.

\section{Sleep study and pSSAS scoring}

All patients then underwent full-night attended PSG at the Sleep Medicine Centre during which the following measurements were continuously recorded: electrooculography, electroencephalography, mental and tibial electromyography, electrocardiography, nasal airflow, pulse oximetry, thoracic and abdominal movements, and snoring intensity (Alice 6, Philips Respironics, the United State). All data was stored on a personal computer and manually analysed in accordance with the published AASM Scoring Manual Version 2.4. According to the guidelines, apnoea was defined when there was a drop in the peak signal excursion by $\geq 90 \%$ of pre-event baseline and the duration of the $\geq 90 \%$ drop in sensor signal was $\geq 10$ seconds, whereas in hypopnea airflow is decreased by more than $30 \%$ for at least 10 seconds, in combination with a reduction in haemoglobin oxygen saturation of at least $3 \%$ or the event is associated with an arousal. All fullnight PSG measurements that lasted more than 6 hours were accepted.

The pSSAS was scored according to Scoring Scheme 1 in the original paper, because this scheme produced the best diagnostic performance in the original study (Table S1).

\section{Statistical analysis}

Normally distributed continuous data are expressed as mean 
Table 1 Demographic and baseline characteristics

\begin{tabular}{|c|c|}
\hline Clinical characteristics & Values \\
\hline Age (years), median (IQR) & $42(14.3)$ \\
\hline \multicolumn{2}{|l|}{ Gender, n (\%) } \\
\hline Female & $22(17.4)$ \\
\hline Male & $104(82.6)$ \\
\hline Height (cm), median (IQR) & $170.58(8.3)$ \\
\hline Weight (kg), median (IQR) & $78(19.5)$ \\
\hline BMI $\left(\mathrm{kg} / \mathrm{m}^{2}\right)$, median (IQR) & $24.2(5.6)$ \\
\hline Neck circumference (cm), median (IQR) & $38.7(4.6)$ \\
\hline \multicolumn{2}{|l|}{$\mathrm{AHI}$ (events/hour), n (\%) } \\
\hline $0 \sim 5$ & $15(11.9)$ \\
\hline $5 \sim 15$ & $30(23.8)$ \\
\hline $15 \sim 30$ & $25(19.8)$ \\
\hline$>30$ & $56(44.5)$ \\
\hline \multicolumn{2}{|l|}{ MiniSO $_{2}, \mathrm{n}(\%)$} \\
\hline$<80 \%$ & $58(46.0)$ \\
\hline$>80 \%$ & $68(54.0)$ \\
\hline ESS (points), median (IQR) & $9.4(6.0)$ \\
\hline BQ (points), median (IQR) & $5.4(3.0)$ \\
\hline pSSAS (points), median (IQR) & $13.4(7.3)$ \\
\hline
\end{tabular}

BMI, body mass index; AHI, apnoea-hypopnea index; ESS, Epworth Sleepiness Scale; BQ, Berlin questionnaire; pSSAS, pictorial Sleepiness and Sleep Apnoea Scale.

$\pm \mathrm{SD}$; categorical data are summarized by their observed frequencies and percentages. We used the BIM SPSS 23 to describe data and analyzed the correlations. Spearman correlations for the relation analysis. The ROC curve was drawn, and the corresponding area under the curve (AUC), sensitivity, specificity, and optimal threshold value were calculated by MedCalc software. All statistical tests were two-sided and the $\mathrm{P}$ value of $<0.05$ was considered as statistically.

\section{Results}

A total of 126 patients were included in the study with the average age being 42 years (range, 22-78 years). Twentytwo were female $(17.5 \%)$ and 104 were male $(82.5 \%)$. The Apnoea-hypopnea index (AHI) scores were classified as mild if between 5 and 15 , moderate between 15 and 30

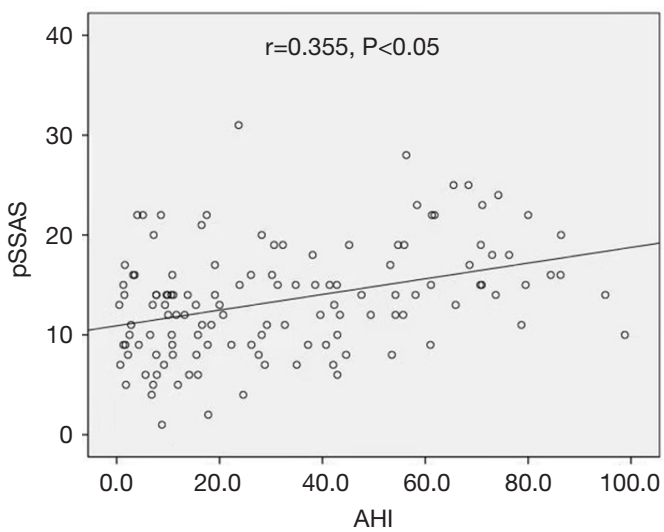

Figure 1 Correlation between AHI and pSSAS: Scatterplot. pSSAS, pictorial Sleepiness and Sleep Apnoea Scale; AHI, apnoeahypopnea index.

and severe if greater than 30 . PSG results showed that 15 patients had an AHI $<5,30$ patients had an AHI in the range 5 to 15,25 patients had an AHI between 15 and 30, and 56 severe cases had an AHI >30. More details are shown in Table 1.

Using these criteria our results showed that AHI had a positive correlation with pSSAS (Figure 1) but the relationship is medium. The relationship between pSSAS and the other scales is shown in Figure 2 from which it can be seen that the pSSAS was moderately correlated with the ESS and BQ.

The comparison of the ability of each score to predict the likelihood of a significant ventilatory problem during sleep is shown in Figures 3-5.

With $\mathrm{AHI}>5$ as the positive diagnostic criterion, the ROC curve is as shown in Figure 3. The AUC of BQ was the highest $(0.730)$, with a certain degree of accuracy, followed by pSSAS and ESS (AUC 0.588, 0.574, respectively) and $\mathrm{P}$ value of $\mathrm{BQ}$ was less than 0.05 which shows that the diagnostic method has diagnostic value (Table 2). When pSSAS was compared with ESS and BQ, P was greater than 0.05 , while BQ, ESS and PSSAS had no significant difference in these diagnostic criteria (Table 3).

With $\mathrm{AHI}>15$ as the positive diagnostic criterion, the ROC curve is as shown above in Figure 4. The AUC of BQ was the highest (0.683), followed by pSSAS and ESS (AUC 0.648 and 0.516 , respectively). Both the $\mathrm{P}$ value of the BQ and pSSAS were less than 0.05 which shows that the diagnostic method had diagnostic value, but low (Table 4). When pSSAS was compared with ESS and BQ, 

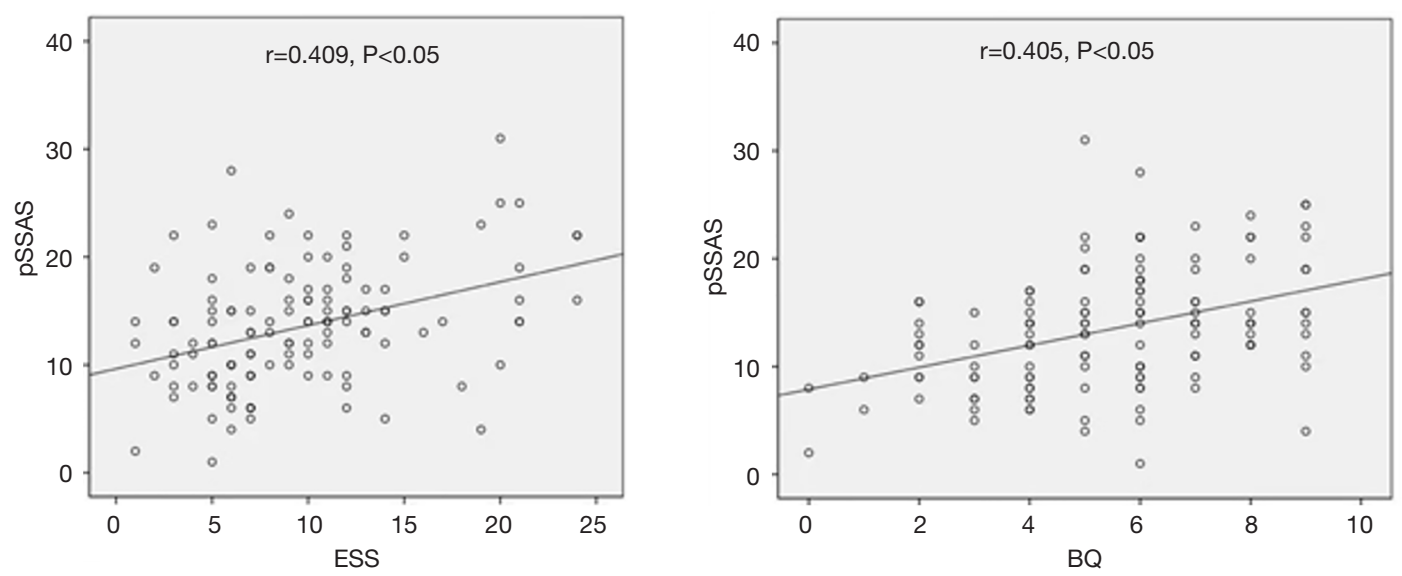

Figure 2 Showing relationship between pSSAS and ESS and BQ. pSSAS was moderately correlated with ESS and BQ ( $\mathrm{r}=0.409$, P $<0.05$; $\mathrm{r}=0.405, \mathrm{P}<0.05)$. pSSAS, pictorial Sleepiness and Sleep Apnoea Scale; ESS, Epworth Sleepiness Scale; BQ, Berlin questionnaire.

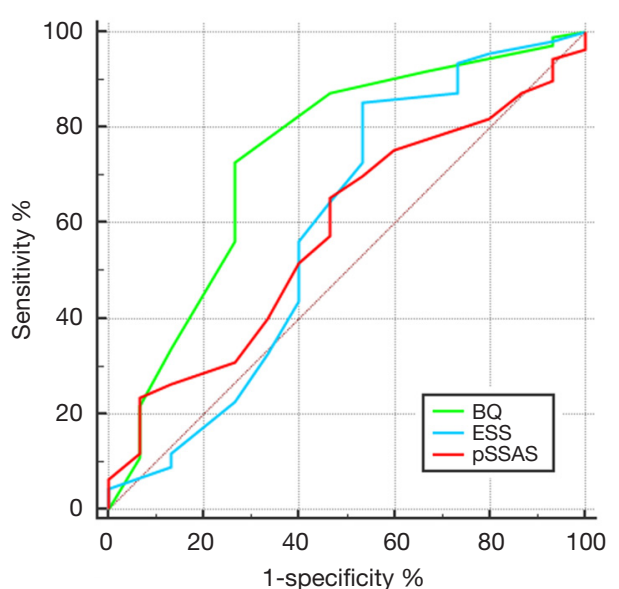

Figure 3 ROC curves showing the sensitivity and specificity for each of the scales when using an AHI $>5$ as the positive diagnostic criteria. BQ, Berlin questionnaire; ESS, Epworth Sleepiness Scale; pSSAS, pictorial Sleepiness and Sleep Apnoea Scale; AHI, Apnoeahypopnea index.

$\mathrm{P}$ was greater than 0.05 , while BQ, ESS and pSSAS had no significant difference (Table 5 ).

With $\mathrm{AHI}>30$ as the positive diagnostic criterion, the ROC curve is as shown above in Figure 5. The AUC of pSSAS was the highest (0.696), followed by BQ and ESS (AUC 0.653 and 0.510 , respectively). Both the $P$ value of the BQ and pSSAS were less than 0.05 which shows that the diagnostic method had diagnostic value and the accuracy of pSSAS is approximate certain (Table 6). In the two comparisons, there was a difference between pSSAS

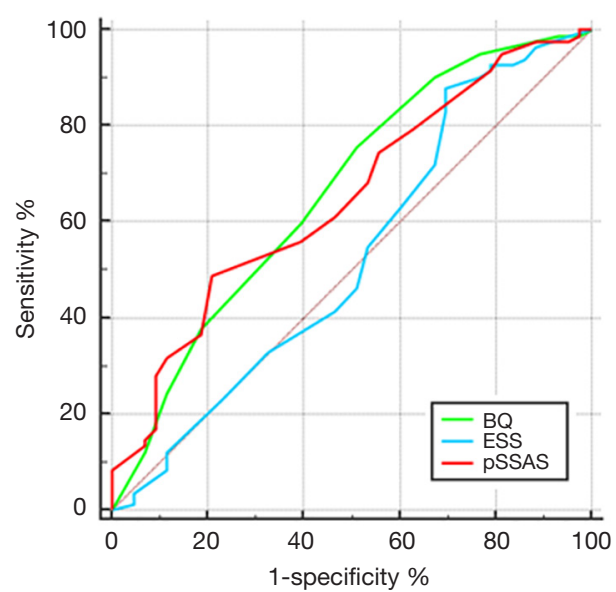

Figure 4 ROC curves showing the sensitivity and specificity for each of the scales when using an $\mathrm{AHI}>15$ as the positive diagnostic criteria. BQ, Berlin questionnaire; ESS, Epworth Sleepiness Scale; pSSAS, pictorial Sleepiness and Sleep Apnoea Scale; AHI, Apnoeahypopnea index.

and ESS $(\mathrm{P}<0.05)$. The diagnostic efficiency of pSSAS was higher than that of ESS, while the $\mathrm{P}$ value of $\mathrm{BQ}$ and pSSAS was more than 0.05 (Table 7).

With $\mathrm{MiniSO}_{2}<80 \%$ as the positive diagnostic criterion, the ROC curve is as shown above in Figure 6. The AUC of pSSAS was the highest (0.736), followed by BQ and ESS (AUC 0.634 and 0.516, respectively). The $P$ value of the BQ and pSSAS were less than 0.05 which shows that the diagnostic method had diagnostic value and the accuracy of pSSAS is certain (Table 8). In the two 


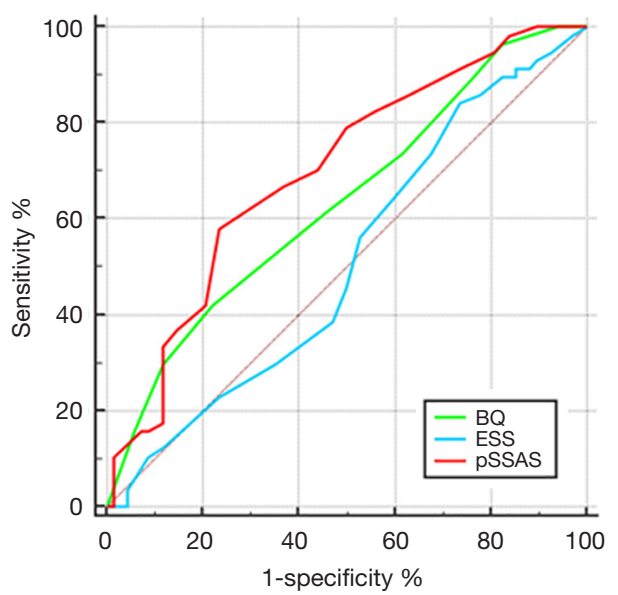

Figure 5 ROC curve showing the sensitivity and specificity for each of the scales when using an $\mathrm{AHI}>30$ as the positive diagnostic criteria. BQ, Berlin questionnaire; ESS, Epworth Sleepiness Scale; pSSAS, pictorial Sleepiness and Sleep Apnoea Scale; AHI, Apnoeahypopnea index. comparisons, there was a difference between pSSAS and ESS $(\mathrm{P}<0.05)$. The diagnostic efficiency of pSSAS was higher than that of ESS, while the $\mathrm{P}$ value of $\mathrm{BQ}$ and pSSAS was less than 0.05 (Table 9).

We also tested the diagnosis accuracy of the pSSAS with a cut-off of 14 points when taking different AHI value as the positive diagnostic criteria (Table 10). The result shows that with the AHI criterion increased, the diagnosis accuracy is getting higher.

\section{Discussion}

\section{OSAS and questionnaires}

OSAS is now widely recognized as a major public health concern with numerous and widespread societal consequences. It is associated with many chronic medical conditions that contribute to poor quality of life, morbidity, and mortality, including hypertension, diabetes, coronary

Table 2 The predictive value of the pSSAS, BQ and ESS when using an AHI >5 as a cut-off for the positive diagnostic criteria

\begin{tabular}{|c|c|c|c|c|c|c|}
\hline Questionnaire & AUC & Sensitivity & Specificity & $\mathrm{P}$ & Youden index & Optimal cut points \\
\hline$B Q$ & $0.730(0.634-0.805)$ & 72.07 & 73.33 & 0.0028 & 0.4541 & 4.5 \\
\hline pSSAS & $0.588(0.497-0.675)$ & 64.86 & 53.33 & 0.2588 & 0.1820 & 11.5 \\
\hline
\end{tabular}

pSSAS, pictorial Sleepiness and Sleep Apnoea Scale; BQ, Berlin questionnaire; ESS, Epworth Sleepiness Scale; AHI, apnoea-hypopnea index; AUC, area under curve.

Table 3 The comparison between pSSAS and BQ, ESS respectively when using an AHI $>5$ as a cut-off for the positive diagnostic criteria

\begin{tabular}{lcc}
\hline pSSAS & ESS & BQ \\
\hline$P$ & 0.9272 & 0.0888 \\
\hline
\end{tabular}

With $\mathrm{AHI}>5$ as the positive diagnostic criterion, the ROC curve is as shown in Figure 3 above. The AUC of BQ was the highest (0.730), with a certain degree of accuracy, followed by pSSAS and ESS (AUC 0.588, 0.574, respectively) and P value of BQ was less than 0.05 which shows that the diagnostic method has diagnostic value. Compared $\mathrm{pSSAS}$ with $\mathrm{ESS}$ and $\mathrm{BQ}$, In the two comparisons, $\mathrm{P}$ was greater than 0.05 , while $B Q$, ESS and pSSAS had no significant difference in these diagnostic criteria. pSSAS, pictorial Sleepiness and Sleep Apnoea Scale; BQ, Berlin questionnaire; ESS, Epworth Sleepiness Scale; AHI, apnoea-hypopnea index; AUC, area under curve.

Table 4 The predictive value of the pSSAS, BQ and ESS when using an AHI $>15$ as a cut-off for the positive diagnostic criteria

\begin{tabular}{|c|c|c|c|c|c|c|}
\hline Questionnaire & AUC & Sensitivity & Specificity & $\mathrm{P}$ & Youden index & Optimal cut points \\
\hline$B Q$ & $0.683(0.594-0.763)$ & 76.54 & 51.11 & 0.0003 & 0.2765 & 4.5 \\
\hline pSSAS & $0.648(0.558-0.731)$ & 48.15 & 77.78 & 0.0036 & 0.2593 & 14.5 \\
\hline
\end{tabular}

pSSAS, pictorial Sleepiness and Sleep Apnoea Scale; BQ, Berlin questionnaire; ESS, Epworth Sleepiness Scale; AHI, apnoea-hypopnea index; AUC, area under curve. 
Table 5 The comparison between pSSAS and BQ, ESS respectively when using an AHI $>15$ as a cut-off for the positive diagnostic criteria

\begin{tabular}{lcc}
\hline pSSAS & ESS & BQ \\
\hline$P$ & 0.1429 & 0.5663 \\
\hline
\end{tabular}

With $\mathrm{AHI}>15$ as the positive diagnostic criterion, the ROC curve is as shown above in Figure 4. The AUC of BQ was the highest (0.683), followed by pSSAS and ESS (AUC 0.648 and 0.516 , respectively). Both the $\mathrm{P}$ value of the BQ and pSSAS were less than 0.05 which shows that the diagnostic method had diagnostic value, but a little low. In the two comparisons, $\mathrm{P}$ was greater than 0.05 , while $B Q$, ESS and pSSAS had no significant difference. pSSAS, pictorial Sleepiness and Sleep Apnoea Scale; BQ, Berlin questionnaire; ESS, Epworth Sleepiness Scale; AHI, apnoea-hypopnea index; AUC, area under curve.

Table 6 The predictive value of the pSSAS, BQ and ESS when using an AHI $>30$ as a cut-off for the positive diagnostic criteria

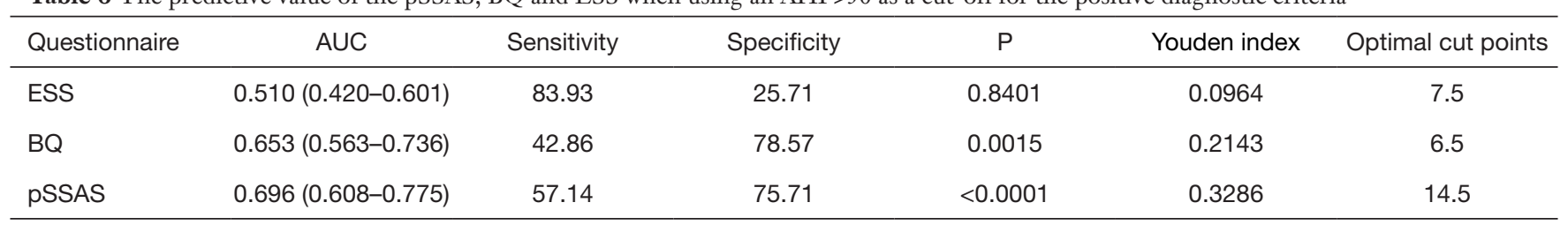

AHI, apnoea-hypopnea index; ESS, Epworth Sleepiness Scale; BQ, Berlin questionnaire; pSSAS, pictorial Sleepiness and Sleep Apnoea Scale; AUC, area under curve.

Table 7 The comparison between pSSAS and BQ, ESS respectively when using an AHI $>30$ as a cut-off for the positive diagnostic criteria

\begin{tabular}{lccc}
\hline pSSAS & ESS & BQ \\
\hline$P$ & 0.028 & 0.4248 \\
\hline
\end{tabular}

With $\mathrm{AHI}>30$ as the positive diagnostic criterion, the ROC curve is as shown above in Figure 5. The AUC of pSSAS was the highest (0.696), followed by BQ and ESS (AUC 0.653 and 0.510 , respectively). Both the $P$ value of the BQ and pSSAS were less than 0.05 which shows that the diagnostic method had diagnostic value and the accuracy of pSSAS is approximate certain. In the two comparisons, there was a difference between pSSAS and ESS $(P<0.05)$. The diagnostic efficiency of pSSAS was higher than that of ESS, while the $P$ value of BQ and pSSAS was more than 0.05. pSSAS, pictorial Sleepiness and Sleep Apnoea Scale; BQ, Berlin questionnaire; ESS, Epworth Sleepiness Scale; AHI, apnoea-hypopnea index; AUC, area under curve.

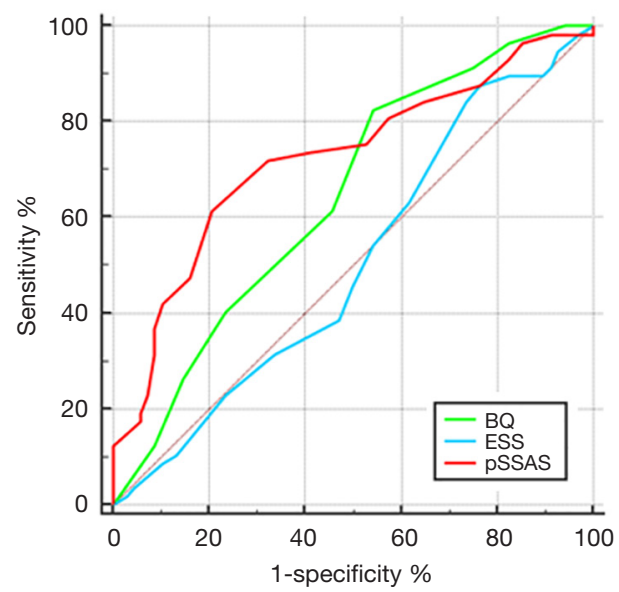

Figure 6 ROC curve showing the sensitivity and specificity for each of the scales when using an $\mathrm{MiniSO}_{2}<80 \%$ as the positive diagnostic criteria. BQ, Berlin questionnaire; ESS, Epworth Sleepiness Scale; pSSAS, pictorial Sleepiness and Sleep Apnoea Scale. artery disease, and diminished cognitive ability. Patients with untreated OSAS have been shown to have increased risk of road traffic incidents and to contribute significantly to increase healthcare costs and resource use (13).

OSAS is reported to be frequently underdiagnosed (14-16), but its prevalence in the population is high (17). The prevalence of moderate to severe OSA is reported to be $6-17 \%$, being as high as $49 \%$ with advanced age (17).

The gold standard for the diagnosis of OSAS is overnight polysomnography performed in sleep laboratories. Polysomnography lasts at least 6 hours and includes a variety of invasive-like recordings such as electroencephalography, electro-oculography, chin electromyography, nasal and oral airflow by thermistors, snoring by microphone, electrocardiogram, pulse oximetry and tibialis anterior electromyogram. These diagnostic procedures are costly and lengthy, which may partly explain 
Table 8 The predictive value of the pSSAS, BQ and ESS when using an $\mathrm{MiniSO}_{2}<80 \%$ as a cut-off for the positive diagnostic criteria

\begin{tabular}{lcccccc}
\hline Questionnaire & AUC & Sensitivity & Specificity & $P$ & Youden index & Optimal cut points \\
\hline ESS & $0.516(0.425-0.606)$ & 12.07 & 76.47 & 0.7577 & 0.1146 & 7.5 \\
BQ & $0.634(0.544-0.718)$ & 81.03 & 45.59 & 0.0061 & 0.2662 & 4.5 \\
pSSAS & $0.736(0.650-0.811)$ & 62.07 & 80.88 & $<0.0001$ & 0.4295 & 14.5 \\
\hline
\end{tabular}

pSSAS, pictorial Sleepiness and Sleep Apnoea Scale; BQ, Berlin questionnaire; ESS, Epworth Sleepiness Scale.

Table 9 The comparison between pSSAS and BQ, ESS respectively when using an $\mathrm{MiniSO}_{2}<80 \%$ as a cut-off for the positive diagnostic criteria

\begin{tabular}{lcc}
\hline pSSAS & ESS & BQ \\
\hline$P$ & $<0.0001$ & 0.0638 \\
\hline
\end{tabular}

With $\mathrm{MiniSO}_{2}<80 \%$ as the positive diagnostic criterion, the ROC curve is as shown above in Figure 6 . The AUC of pSSAS was the highest (0.736), followed by BQ and ESS (AUC 0.634 and 0.516 , respectively). The $P$ value of the BQ and pSSAS were less than 0.05 which shows that the diagnostic method had diagnostic value and the accuracy of pSSAS is certain. In the two comparisons, there was a difference between pSSAS and ESS $(P<0.05)$. The diagnostic efficiency of pSSAS was higher than that of ESS, while the $P$ value of $B Q$ and $p S S A S$ was less than 0.05. pSSAS, pictorial Sleepiness and Sleep Apnoea Scale; BQ, Berlin questionnaire; ESS, Epworth Sleepiness Scale.

Table 10 Comparing the diagnostic accuracy of different AHI criteria which use a pSSAS cut-off of 14 points

\begin{tabular}{lcccccc}
\hline Criterion and cut-off & Sensitivity & Specificity & LR+ & LR- & Youden index & Diagnosis accuracy \\
\hline AHI criterion $>5$, pSSAS cut-off $=14$ & $39.6 \%$ & $66.6 \%$ & 1.19 & 0.91 & 0.062 & $42.9 \%$ \\
AHI criterion $>15$, pSSAS cut-off $=14$ & $48.8 \%$ & $79.5 \%$ & 2.38 & 0.64 & 0.283 & $59.5 \%$ \\
AHI criterion $>30$, pSSAS cut-off $=14$ & $57.9 \%$ & $76.8 \%$ & 2.50 & 0.55 & 0.347 & $68.3 \%$ \\
\hline
\end{tabular}

LR+, likelihood ratio positive; LR-, likelihood ratio negative.

why $82 \%$ of men and $92 \%$ of women in the USA who are likely to have moderate or severe OSAS have yet to be diagnosed $(18,19)$.

An ideal screening tool would be easy to complete by all patients and have a sensitivity and specificity that neither suggested a diagnosis in those without the condition or missed cases requiring investigation and treatment. Such a tool then permits those with a high likelihood of the condition to have simple confirmatory tests and institution of appropriate management leaving complex investigations for those with equivocal scores or comorbidities. Screening tools such as the BQ, STOP-BANG questionnaire (SBQ), and STOP questionnaire have been widely promoted for detecting OSA. The Epworth sleepiness scale (ESS), which was originally designed to assess the risk of daytime sleepiness, has been extensively used in the community prior to specialist referral in many countries as part of the screening process (4), but has not been shown to be a satisfactory screening test by itself.

The recently published European Respiratory Society statement on sleep apnoea, sleepiness and driving risk (20) concludes that certainly with regards to driving none of the existing screening questionnaires is satisfactory and calls for more research on new questionnaires. In the general population, extreme variation can be found in the sensitivities and specificities for all established questionnaires (ESS sensitivity 18-85\%, specificity 22-98\% (21-23); BQ sensitivity 40-97\%, specificity 6-100\% (24-30); STOP sensitivity 33-98\%, specificity 10-95\% (31); STOPBang sensitivity 0-100\%, specificity 0-100\% (25,31-33), even taking into account the cut-off thresholds for OSA (AHI $\geq 5$ events/h, AHI $\geq 15$ events/h, AHI $\geq 30$ events/h).

Sensitivity was highest in sleep clinic samples for all questionnaires, while the ESS showed an overall poor predictive value. In clinical population samples (i.e., patients admitted or followed for disease other than OSAS), on average the $\mathrm{BQ}$ showed a higher sensitivity compared to the STOP-Bang. Clearly, the data indicate that questionnaires neither reliably rule in nor rule out OSA (20).

Therefore, developing a new questionnaire with higher 
sensitivities and specificities is one of the future research priorities of OSAS.

\section{pSSAS}

Written questionnaires can be difficult for some patients to complete, and studies have shown that reduced literacy is common in patients attending a sleep clinic (9). In addition to the effect of increased sleepiness, verbal episodic memory can be impaired in patients with OSAS (34). This led to the development of a pictorial Epworth Sleepiness Scale (pESS) in 2011, and the same team further developed a pSSAS questionnaire that consists of eight items to be used for OSAS screening, four common to the pESS and four related to body morphology, blood pressure and witnessed apnoea (35-37).

Its simple structure, scoring rules and the use of pictograms make it an alternative to more complex and long questionnaires in clinical practice and in patients with reduced literacy. The validation study has shown good internal consistency, repeatability and construct validity when compared with established tools for the diagnosis of excessive daytime sleepiness (EDS) and OSAS (10).

Pictures with words have been shown to enhance the understanding and translation of medical information, and a pictorial translation of the ESS produces scores comparable with the traditional ESS and may be a suitable alternative for those with normal or diminished literacy (35). To elicit the best possible understanding, combining both pictures and words to maximize intended meaning is important and necessary (38-41). Another type of pictorial sleepiness scale has recently been reported for use amongst indigenous Australian adults (42).

In this report we have demonstrated that a pictorial sleep apnoea screening score which was originally validated in a British and a Swiss population (10) appears to be as useful in a Chinese population of patients with suspected OSAS. This confirms the suggestion from another recent paper from China (11) that morphological risk factors such as neck circumference and BMI that apply in European populations are also important in a Chinese population. The results show that the pSSAS is as effective as the BQ in predicting those likely to have OSAS and that the pSSAS is better at predicting those who have severe OSA (AHI >30).

Proving that a new test performs in a similar fashion to existing tests is a worthwhile exercise and equivalence increases choice but does the pSSAS have advantages? Administering some questionnaires can be very time consuming for health professionals and the pSSAS has the potential advantage that it is pictorial. Our previous work has shown that a third of new patients make quantifiable errors when completing written sleepiness scores and a preference for a pESS was shown by $55 \%$ of users who found it easier to complete than the worded version (35). It is not possible for health professionals to predict which patients have impaired health literacy $(8,43)$ and formal assessment is both time consuming and potentially distressing to patients. Universal precautions should therefore be applied with the simplest tools offered to all. The pSSAS can be self-completed but in our experience some patients benefit from assistance with the risk questions (Questions 5, 6, 7 and 8). Problems associated with health literacy (and numeracy) lead to poorer health outcomes which can be expensive for health care systems (44) and yet literacy sensitive approaches have shown that such approaches in several respiratory conditions enable those with impairment to develop equal outcomes to those not so affected $(45,46)$.

\section{BMI, gender and OSA}

Among Chinese, OSA occurs at lower body mass indices (BMIs) than in Caucasians, suggesting that craniofacial differences within the Chinese population play a major role. In Singapore (47), a multiethnic nation, Chinese had a significantly higher prevalence of OSA [apnoeahypopnea index (AHI) of $\geq 15$ events/h] despite having a lower BMI than both Indians and Malays. This is supported by the Multi-Ethnic Study of Atherosclerosis (MESA) Sleep Cohort (48). As to why Chinese may have OSA at a lower BMI, a study (49) comparing Caucasians in Australia to Chinese in Hong Kong (matched for OSA severity) found Caucasians to be more overweight with larger tongues whereas Chinese exhibited more craniofacial bony restriction. Our study also shows that Chinese OSA patients (confirmed by PSG) have a lower BMI when compared with Caucasians. We also should pay more attention to the different cut-offs of obesity between the different populations. Chang's study demonstrated that Chinese Taiwan subjects had a relatively lower BMI but a higher percent body fat than Caucasians. In general, the newly proposed Asia-Pacific BMI cut-offs for overweight $\left(\geq 23 \mathrm{~kg} / \mathrm{m}^{2}\right)$ and obesity $\left(\geq 25 \mathrm{~kg} / \mathrm{m}^{2}\right)$ may be acceptable to both male and female Chinese Taiwan subjects (50). However, obesity in China is progressively increasing with the development of the economy and the concomitant 
changes in Chinese life style. Future research focusing on the differences between obese and non-obese patients may provide useful targets for specific treatments for each of these groups.

Besides these risk factors such as obesity, increased age, cigarette smoking, and craniofacial abnormalities, male gender is recognized as an independent risk factor for the development of the syndrome. Consequently, and unsurprisingly, OSAS has thus been considered a male disease, with male: female ratios ranging from 3:1 to 5:1 in the general population and from $8: 1$ to $10: 1$ in selected clinical populations (51). In our study, the male: female ration is 4.7 which is in the range of the above studies. However, according to our experience, female OSA patients are underdiagnosed in medical practice. Failure to recognize the distinct clinical presentation and sex-specific differences in sleep studies may lead to underdiagnosis or misdiagnosis of OSAS in females $(52,53)$. Future studies should focus on the specific female OSA characteristics.

\section{Conclusions}

We have demonstrated that the use of a literacy sensitive tool in risk stratification in suspected OSAS produces results equivalent to, or indeed better than more complicated written questionnaires which patients may need significant assistance to complete without errors.

\section{Acknowledgments}

The original development of the pSSAS was undertaken by Dr. Ramesh Ghiassi as part of her $\mathrm{PhD}$ thesis supervised by MRP, and submitted to Imperial College London in 2014. The authors are grateful to Mary S. M. Ip for her important comments during manuscript preparation.

Funding: This study was financially supported by the following two grants: Sanming Project of Medicine in Shenzhen, China, "Integrated Airways Disease Team led by Professor Kian Fan Chung from Imperial College London" (No. SZSM201612096); High Level-hospital Program, Health Commission of Guangdong Province, China (No. HKUSZH201901010).

\section{Footnote}

Reporting Checklist: The authors have completed the STARD reporting checklist. Available at https://dx.doi. org/10.21037/jtd-20-2152
Data Sharing Statement: Available at https://dx.doi. org/10.21037/jtd-20-2152

Conflicts of Interest: All authors have completed the ICMJE uniform disclosure form (available at https://dx.doi. org/10.21037/jtd-20-2152). All authors report funding from the Sanming Project of Medicine in Shenzhen, China and the High Level- hospital Program, Health Commission of Guangdong Province, China.

Ethical Statement: The authors are accountable for all aspects of the work in ensuring that questions related to the accuracy or integrity of any part of the work are appropriately investigated and resolved. The study was conducted in accordance with the Declaration of Helsinki (as revised in 2013). This study has documented review and approval from the University of Hong Kong-Shenzhen Hospital Institutional Review Board (approval number [2019]092). Written informed consent have been provided by the participants.

Open Access Statement: This is an Open Access article distributed in accordance with the Creative Commons Attribution-NonCommercial-NoDerivs 4.0 International License (CC BY-NC-ND 4.0), which permits the noncommercial replication and distribution of the article with the strict proviso that no changes or edits are made and the original work is properly cited (including links to both the formal publication through the relevant DOI and the license). See: https://creativecommons.org/licenses/by-nc-nd/4.0/.

\section{References}

1. Corral J, Sánchez-Quiroga MÁ, Carmona-Bernal C, et al. Conventional Polysomnography Is Not Necessary for the Management of Most Patients with Suspected Obstructive Sleep Apnea. Noninferiority, Randomized Controlled Trial. Am J Respir Crit Care Med 2017;196:1181-90.

2. Hui DS, Ng SS, To KW, et al. A randomized controlled trial of an ambulatory approach versus the hospital-based approach in managing suspected obstructive sleep apnea syndrome. Sci Rep 2017;8:45901.

3. Stradling J. Obstructive sleep apnoea: is it moving into primary care? Br J Gen Pract 2016;66:e149-51.

4. Johns MW. A new method for measuring daytime sleepiness: the Epworth sleepiness scale. Sleep 1991;14:540-5.

5. Chung F, Subramanyam R, Liao P, et al. High STOP- 
Bang score indicates a high probability of obstructive sleep apnoea. Br J Anaesth 2012;108:768-75.

6. Netzer NC, Stoohs RA, Netzer CM, et al. Using the Berlin Questionnaire to identify patients at risk for the sleep apnea syndrome. Ann Intern Med 1999;131:485-91.

7. Mollayeva T, Thurairajah P, Burton K, et al. The Pittsburgh sleep quality index as a screening tool for sleep dysfunction in clinical and non-clinical samples: A systematic review and meta-analysis. Sleep Med Rev 2016;25:52-73.

8. Storms H, Aertgeerts B, Vandenabeele F, et al. General practitioners' predictions of their own patients' health literacy: a cross-sectional study in Belgium. BMJ Open 2019;9:e029357.

9. Ghiassi R, Partridge MR. Health literacy and sleep apnoea. Thorax 2011;66:180.

10. Edelmann C, Ghiassi R, Vogt DR, et al. A pictorial Sleepiness and Sleep Apnoea Scale to recognize individuals with high risk for obstructive sleep apnea syndrome. Nat Sci Sleep 2017;9:253-65.

11. Peng M, Chen R, Cheng J, et al. Application value of the NoSAS score for screening sleep-disordered breathing. J Thorac Dis 2018;10:4774-81.

12. Peng LL, Li JR, Sun JJ, et al. Reliability and validity of the simplified Chinese version of Epworth sleepiness scale. Zhonghua Er Bi Yan Hou Tou Jing Wai Ke Za Zhi 2011;46:44-9.

13. Leger D, Bayon V, Laaban JP, et al. Impact of sleep apnea on economics. Sleep Med Rev 2012;16:455-62.

14. Fuhrman C, Fleury B, Nguyên XL, et al. Symptoms of sleep apnea syndrome: high prevalence and underdiagnosis in the French population. Sleep Med 2012;13:852-8.

15. Kapur V, Strohl KP, Redline S, et al. Underdiagnosis of sleep apnea syndrome in U.S. communities. Sleep Breath 2002;6:49-54.

16. Simpson L, Hillman DR, Cooper MN, et al. High prevalence of undiagnosed obstructive sleep apnoea in the general population and methods for screening for representative controls. Sleep Breath 2013;17:967-73.

17. Senaratna CV, Perret JL, Lodge CJ, et al. Prevalence of obstructive sleep apnea in the general population: A systematic review. Sleep Med Rev 2017;34:70-81.

18. Young T, Palta M, Dempsey J, et al. The occurrence of sleep-disordered breathing among middle-aged adults. N Engl J Med 1993;328:1230-5.

19. Gibson GJ. Obstructive sleep apnoea syndrome: underestimated and undertreated. Br Med Bull 2005;72:49-65.
20. Bonsignore MR, Randerath W, Schiza S, et al. European Respiratory Society statement on sleep apnoea, sleepiness and driving risk. Eur Respir J 2021;57:2001272.

21. Sil A, Barr G. Assessment of predictive ability of Epworth scoring in screening of patients with sleep apnoea. J Laryngol Otol 2012;126:372-9.

22. Bhat S, Upadhyay H, DeBari VA, et al. The utility of patient-completed and partner-completed Epworth Sleepiness Scale scores in the evaluation of obstructive sleep apnea. Sleep Breath 2016;20:1347-54.

23. Nishiyama T, Mizuno T, Kojima M, et al. Criterion validity of the Pittsburgh Sleep Quality Index and Epworth Sleepiness Scale for the diagnosis of sleep disorders. Sleep Med 2014;15:422-9.

24. Ramachandran SK, Josephs LA. A meta-analysis of clinical screening tests for obstructive sleep apnea. Anesthesiology 2009;110:928-39.

25. Westlake K, Plihalova A, Pretl M, et al. Screening for obstructive sleep apnea syndrome in patients with type 2 diabetes mellitus: a prospective study on sensitivity of Berlin and STOP-Bang questionnaires. Sleep Med 2016;26:71-6.

26. Marti-Soler H, Hirotsu C, Marques-Vidal P, et al. The NoSAS score for screening of sleep-disordered breathing: a derivation and validation study. Lancet Respir Med 2016;4:742-8.

27. Singh A, Prasad R, Garg R, et al. A study to estimate prevalence and risk factors of Obstructive Sleep Apnoea Syndrome in a semi-urban Indian population. Monaldi Arch Chest Dis 2017;87:773.

28. Migacz E, Wichniak A, Kukwa W. Are questionnaires reliable in diagnosing sleep-disordered breathing in university students? J Laryngol Otol 2017;131:965-71.

29. Abrishami A, Khajehdehi A, Chung F. A systematic review of screening questionnaires for obstructive sleep apnea. Can J Anaesth 2010;57:423-38.

30. Senaratna CV, Perret JL, Matheson MC, et al. Validity of the Berlin questionnaire in detecting obstructive sleep apnea: A systematic review and meta-analysis. Sleep Med Rev 2017;36:116-24.

31. Chiu HY, Chen PY, Chuang LP, et al. Diagnostic accuracy of the Berlin questionnaire, STOP-BANG, STOP, and Epworth sleepiness scale in detecting obstructive sleep apnea: A bivariate meta-analysis. Sleep Med Rev 2017;36:57-70.

32. Nagappa M, Liao P, Wong J, et al. Validation of the STOP-Bang Questionnaire as a Screening Tool for Obstructive Sleep Apnea among Different Populations: 
A Systematic Review and Meta-Analysis. PLoS One 2015;10:e0143697.

33. Gantner D, Ge JY, Li LH, et al. Diagnostic accuracy of a questionnaire and simple home monitoring device in detecting obstructive sleep apnoea in a Chinese population at high cardiovascular risk. Respirology 2010;15:952-60.

34. Twigg GL, Papaioannou I, Jackson M, et al. Obstructive sleep apnea syndrome is associated with deficits in verbal but not visual memory. Am J Respir Crit Care Med 2010;182:98-103.

35. Ghiassi R, Murphy K, Cummin AR, et al. Developing a pictorial Epworth Sleepiness Scale. Thorax 2011;66:97-100.

36. Drakatos P, Ghiassi R, Jarrold I, et al. The use of an online pictorial Epworth Sleepiness Scale in the assessment of age and gender specific differences in excessive daytime sleepiness. J Thorac Dis 2015;7:897-902.

37. Karim A, Arora VK, Gupta MB. Emerging applications: Screening OSA by Modified Pictorial Epworth Sleepiness Scale in Indian subjects. Indian J Tuberc 2015;62:222-5.

38. Houts PS, Bachrach R, Witmer JT, et al. Using pictographs to enhance recall of spoken medical instructions. Patient Educ Couns 1998;35:83-8.

39. Houts PS, Witmer JT, Egeth HE, et al. Using pictographs to enhance recall of spoken medical instructions II. Patient Educ Couns 2001;43:231-42.

40. Houts PS, Doak CC, Doak LG, et al. The role of pictures in improving health communication: a review of research on attention, comprehension, recall, and adherence.

Patient Educ Couns 2006;61:173-90.

41. Katz MG, Kripalani S, Weiss BD. Use of pictorial aids in medication instructions: a review of the literature. Am J Health Syst Pharm 2006;63:2391-7.

42. Benn E, Wirth H, Short T, et al. The Top End Sleepiness Scale (TESS): A New Tool to Assess Subjective Daytime Sleepiness Among Indigenous Australian Adults. Nat Sci Sleep 2021;13:315-28.

43. Rogers ES, Wallace LS, Weiss BD. Misperceptions of medical understanding in low-literacy patients:

Cite this article as: Ji Y, Wang H, Liu M, Partridge MR. Use of the pictorial Sleepiness and Sleep Apnoea Scale in Chinese patients with suspected obstructive sleep apnoea syndrome. J Thorac Dis 2021;13(10):6071-6081. doi: 10.21037/jtd-20-2152 implications for cancer prevention. Cancer Control 2006;13:225-9.

44. Haun JN, Patel NR, French DD, et al. Association between health literacy and medical care costs in an integrated healthcare system: a regional population based study. BMC Health Serv Res 2015;15:249.

45. Paasche-Orlow MK, Riekert KA, Bilderback A, et al. Tailored education may reduce health literacy disparities in asthma self-management. Am J Respir Crit Care Med 2005;172:980-6.

46. Kiser K, Jonas D, Warner Z, et al. A randomized controlled trial of a literacy-sensitive self-management intervention for chronic obstructive pulmonary disease patients. J Gen Intern Med 2012;27:190-5.

47. Tan A, Cheung YY, Yin J, et al. Prevalence of sleepdisordered breathing in a multiethnic Asian population in Singapore: A community-based study. Respirology 2016;21:943-50.

48. Chen X, Wang R, Zee P, et al. Racial/Ethnic Differences in Sleep Disturbances: The Multi-Ethnic Study of Atherosclerosis (MESA). Sleep 2015;38:877-88.

49. Lee RW, Vasudavan S, Hui DS, et al. Differences in craniofacial structures and obesity in Caucasian and Chinese patients with obstructive sleep apnea. Sleep 2010;33:1075-80.

50. Chang CJ, Wu CH, Chang CS, et al. Low body mass index but high percent body fat in Taiwanese subjects: implications of obesity cutoffs. Int J Obes Relat Metab Disord 2003;27:253-9.

51. Wimms A, Woehrle H, Ketheeswaran S, et al. Obstructive Sleep Apnea in Women: Specific Issues and Interventions. Biomed Res Int 2016;2016:1764837.

52. Kapsimalis F, Kryger MH. Gender and obstructive sleep apnea syndrome, part 1: Clinical features. Sleep 2002;25:412-9.

53. Sforza E, Chouchou F, Collet P, et al. Sex differences in obstructive sleep apnoea in an elderly French population. Eur Respir J 2011;37:1137-43. 


\section{Supplementary}

Patient name

Hospital Number

Date of Birth

Today's Date

\section{The pictorial Sleepiness and Sleep Apnoea Scale (pSSAS)}

Tick $\checkmark$ one box only in each different situation that best represents you.

How likely are you to doze off or fall asleep in the following situations?

1. Sitting and Reading

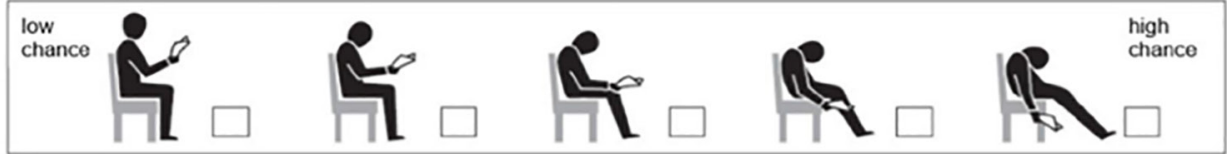

2. Watching TV or listening to the radio

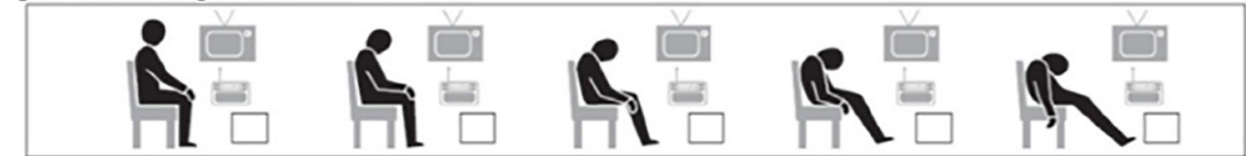

3. Sitting quietly after a meal

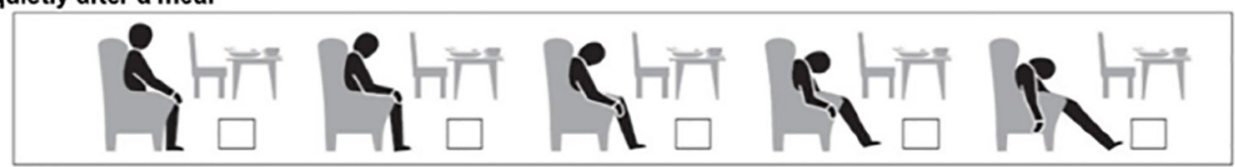

4. As a passenger in a car or bus

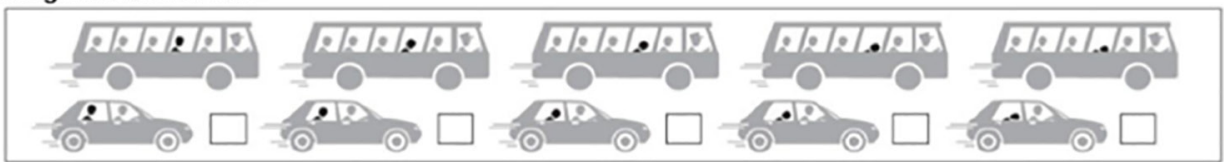

5. Body size

Now please tick $\sqrt{ }$ one box only in each series of pictures that best represents you.

6. Neck size
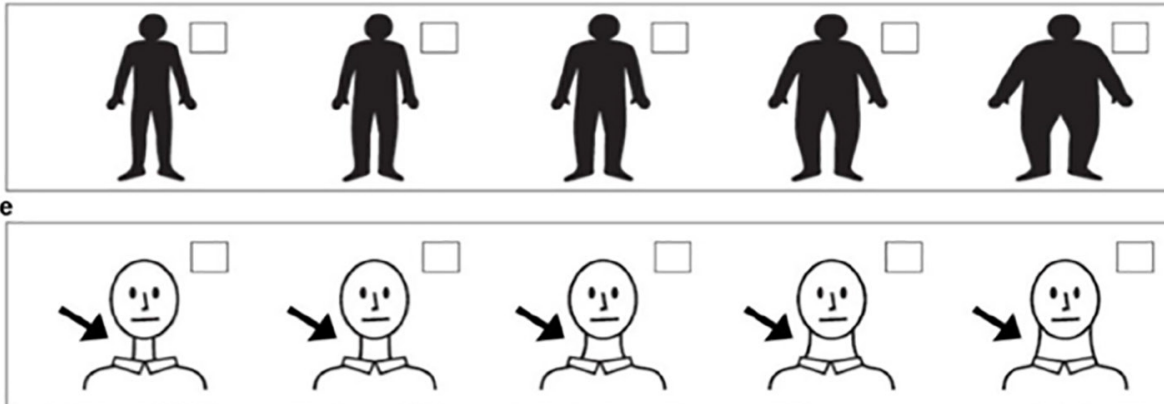

7. Blood pressure: normal
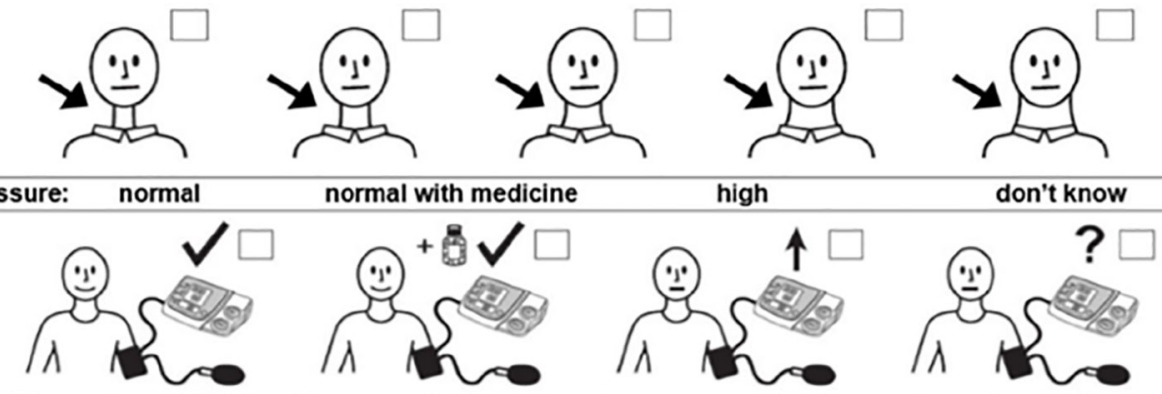

8. Breathing when asleep:

breathing normally when asleep

stopping breathing when asleep don't know
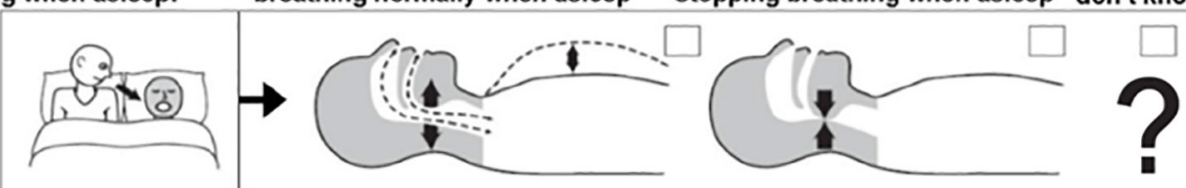

Figure S1 English version of the pSSAS questionnaire. Permission given by Professor Martyn R. Partridge. 
Table S1 pSSAS scoring scheme

\begin{tabular}{ll}
\hline pSSAS item & Scoring \\
\hline Q1-4: Daytime sleepiness & $0,1,2,3$ and 4 points with increasing score indicating increasing daytime sleepiness \\
Q5: Body size & $0,1,2,3,4$ points with increasing score indicating increasing body size \\
Q6: Neck size & $0,1,2,3,4$ points with increasing score indicating increasing neck size \\
Q7: Blood pressure & Normal $=0$ point, normal on medication or high BP $=4$ points, don't know $=0$ point \\
Q8: Witnessed apnoea & Normal breathing $=0$ points, breathing pauses occur $=4$ points, don't know $=0$ points \\
\hline
\end{tabular}

